\title{
The removal of 2,4-dichlorophenol under visible light irradiation by silver indium sulfide nanoparticles synthesized by microwave
}

\author{
Azadeh Tadjarodi*, Amir Hossein Cheshme khavar and Mina Imani
}

Research Laboratory of Inorganic Materials Synthesis, Department of Chemistry, Iran University of Science and Technology, Narmak, Tehran, 1684613114, Iran

\begin{tabular}{l}
\hline C H R O N I C L E \\
\hline Article history: \\
Received October 28, 2012 \\
Received in Revised form \\
December 6, 2012 \\
Accepted 27 February 2013 \\
Available online \\
1 March 2013 \\
\hline Keywords: \\
AgInS2 \\
Nanoparticles \\
Photodegradation \\
Dichlorophenol \\
Microwave
\end{tabular}

\begin{abstract}
A B S T R A C T
Silver indium sulfide $\left(\mathrm{AgInS}_{2}\right)$ nanoparticles were synthesized by microwave method. These nanopartricles were characterized by FT-IR, XRD, DRS, SEM and TEM techniques. The band gap energy of $1.96 \mathrm{eV}$ was determined by UV-Vis diffuse reflection spectrum (DRS). The photocatalytic activity was studied by photodegradation reaction of 2,4-dichlorophenol $(2,4-$ DCP) under visible light irradiation. The influence of initial concentration, initial solution $\mathrm{pH}$ on the degradation percentage of 2,4-DCP and also, the kinetics of photodegradation were investigated. The removal efficiency up to $95 \%$ proved the superior capability of $\mathrm{AgInS}_{2}$ (AIS) nanoparticles for water purification.
\end{abstract}

(C) 2013 Growing Science Ltd. All rights reserved.

\section{Introduction}

The chlorophenols are extensively used as fungicide, herbicide, wood preservative, dyes and drugs $^{1-2}$. These materials have been listed as toxic pollutants by the various environmental protection agencies such as the United States Environmental Protection Agency ${ }^{3}$. The increase of discharging these materials from the industrial and domestic activities into the environment leads to enhance the waters pollution. Being toxic and harmful for environment and living organisms and the shortage of water sources, these pollutants should be removed. Nowadays, the variety of photocatalysts is employed for solving the serious environmental and pollution challenges ${ }^{3-5}$. In fact, the use of photocatalysis technology for destruction of the organic pollutants in water is an interesting method,

* Corresponding author. Tel: +98 2177240359

E-mail addresses: tajarodi@iust.ac.ir (A. Tadjarodi)

C 2013 Growing Science Ltd. All rights reserved. doi: $10.5267 /$ j.ccl.2013.03.001 
which can be replaced to the traditional methods ${ }^{6-10}$. In this technique, the active species such as $\mathrm{OH}^{*}$, $\mathrm{O}_{2}{ }^{--}$and hole $\left(\mathrm{h}^{+}\right)$are generated by light energy and performed the degradation of organic pollutants ${ }^{10-11}$. Although, the semiconductors such as $\mathrm{TiO}_{2}$ and $\mathrm{ZnO}$ with a wide band gap of 3.23-3.37 eV are the best operational available, these are only activated under UV light irradiation, which accounts for around $5 \%$ of the total solar energy. Therefore, a decreased band gap can extend the photocatalytic application from UV light region into the visible light range ${ }^{12-13}$.

Many studies have been carried out to exploit the new semiconductor materials with a narrow band gap adapted to the visible light domain. $\mathrm{AgInS}_{2}$ is a ternary chalchogenide with direct band gap about $1.86-2.03 \mathrm{eV}$ and high extinction coefficient in visible light region ${ }^{14-19}$. Thus, this compound can be introduced as a favorable material for photovoltaic and photocatalytic applications. There are several routes for preparation of $\mathrm{AgInS}_{2}$ nanoparticles ${ }^{10,14-16,18,20-26}$. One of these methods is microwave irradiation process.

In the present study, $\mathrm{AgInS}_{2}$ nanoparticles with the pure orthorhombic phase were synthesized by the microwave method. The photocatalytic activity of $\mathrm{AgInS}_{2}$ nanoparticles was investigated for degradation of 2,4-dichlorophenol (2,4-DCP) under visible light irradiation.

\section{Results and Discussion}

\subsection{Structural and morphological study}

Fig. 1 shows the XRD pattern of the synthesized AIS sample. All of the diffraction peaks confirm the crystalline phase of orthorhombic $\mathrm{AgInS}_{2}$ (JCPDS No. 25-1328). In addition, no characteristic peaks of the impurities are observed in this pattern, implying that a pure orthorhombic phase of $\mathrm{AgInS}_{2}$ has been synthesized.
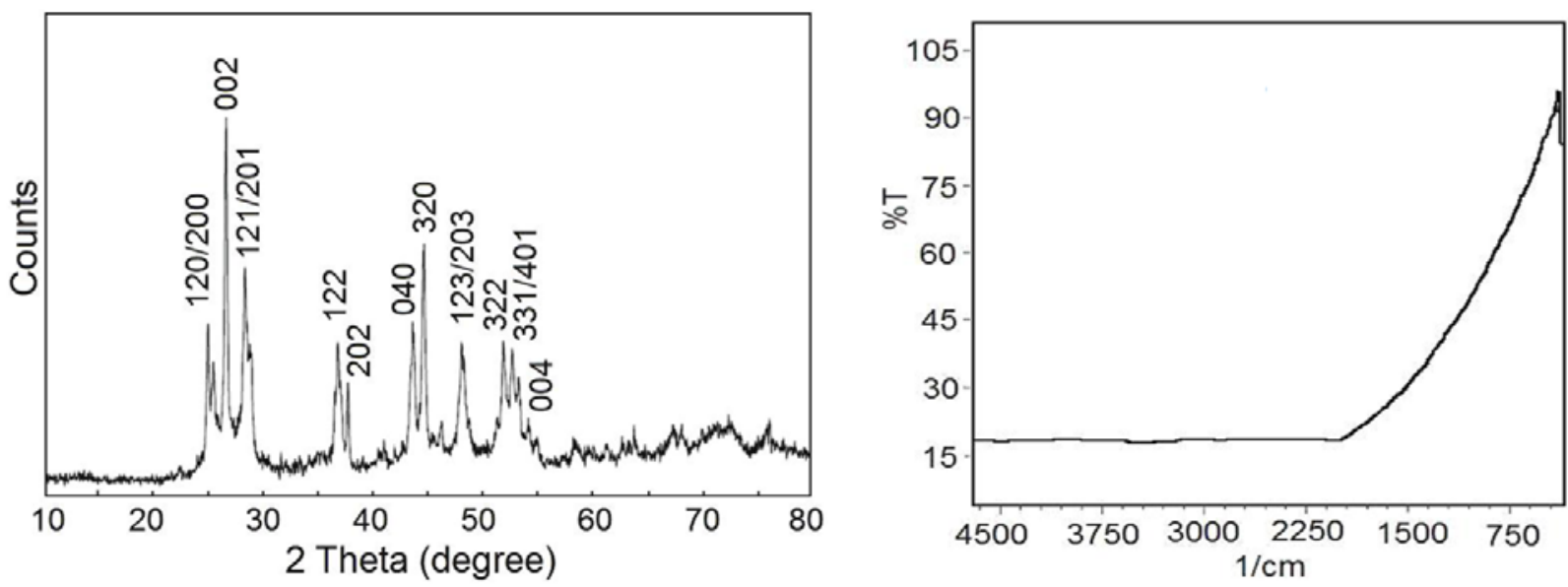

Fig. 1. XRD pattern of $\mathrm{AgInS}_{2}$ nanoparticles

Fig. 2. FT-IR spectrum of $A g I n S_{2}$ nanoparticles

FT-IR spectrum shown in Fig. 2 confirmed the purity of the obtained product. There are no peaks in this spectrum, which represents the removal of organic sections via washing with ethanol and distilled water. The morphology of as-synthesized product was studied by SEM and TEM techniques. The SEM images revealed the uniform particulate morphology with the average particle size of 70 nm (Figs. 3a and b).

The TEM image shown in Fig. 3c further confirmed the results of SEM technique. The average particle size was estimated using a microstructure measurement program and Minitab statistical software ${ }^{27,28}$. The histogram of the particles size distribution of the obtained sample has been illustrated in Fig. 3d. 

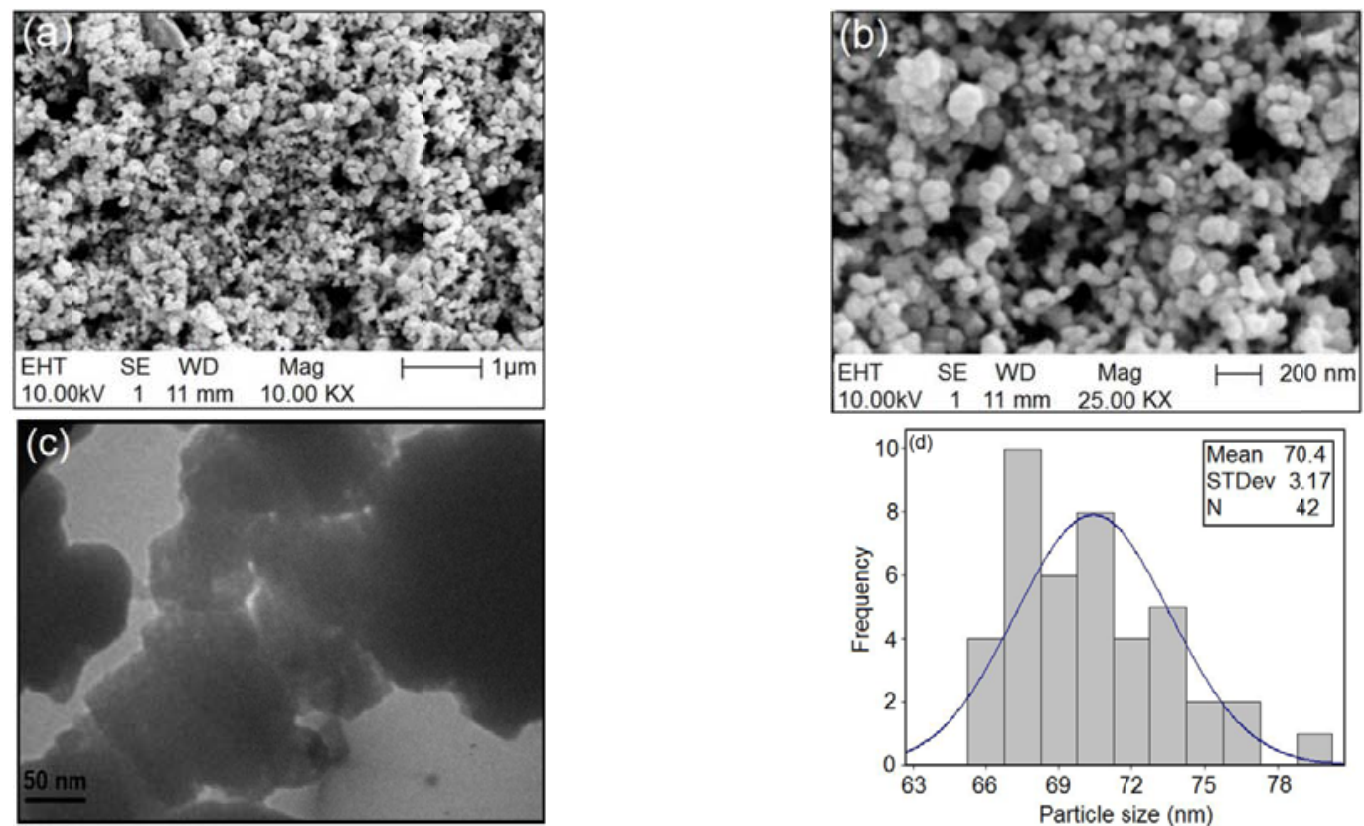

Fig. 3. SEM images (a-b), TEM image (c) and the statistical graph of particle size distribution (d) of AgInS $_{2}$ nanoparticles

The nitrogen adsorption and desorption experiments were performed for determining the surface area of these nanoparticles. A distinct hysteresis loop recorded at the range of $0<\mathrm{P} / \mathrm{P}_{0}<1$ indicated the category of type V (Fig. 4). The Brunauer-Emmett-Teller (BET) surface area about $5.9 \mathrm{~m}^{2} \mathrm{~g}^{-1}$ was calculated for synthesized product. The Barrett-Joyner-Halenda (BJH) model using desorption branch of the nitrogen isotherm was applied to evaluate the pore size distribution of AIS nanoparticles, which was centered at $1.64 \mathrm{~nm}$ (inserted in the inset of Fig. 4). It is an acceptable value for AIS nanoparticles in comparison with the other reports ${ }^{10}$, which can be referred to the presence of active sites on the surface of AIS nanoparticles.

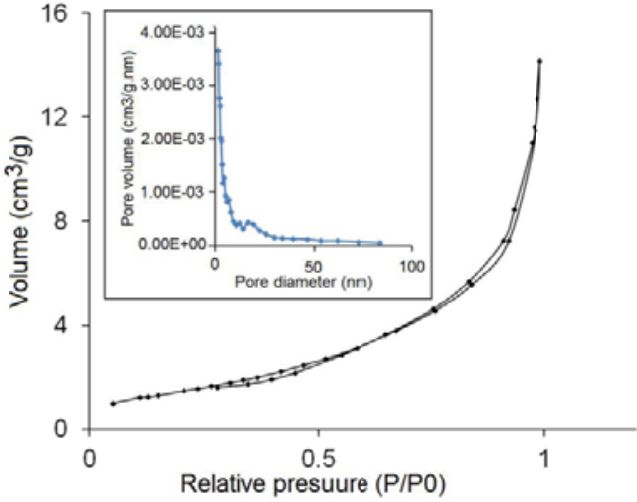

Fig. 4. Nitrogen adsorption $(\diamond)$ and desorption $(\boldsymbol{\Delta})$ isotherms for the AIS nanoparticles. The inset shows BJH plot of this product

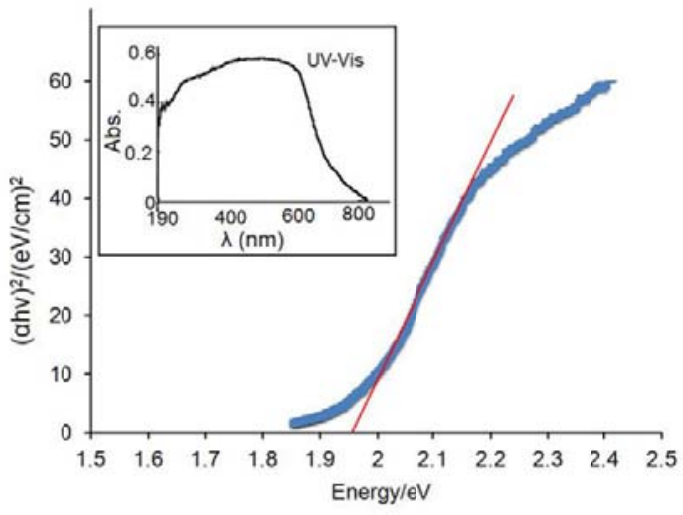

Fig. 5. The plot of $(\alpha h v)^{2} v s$. hv (eV) for determining the direct band gap energy of the prepared $\mathrm{AgInS}_{2}$ nanoparticles with UV-Vis spectrum in the inset

\subsection{The optical property}

The optical property of the AIS nanoparticles was investigated by the band gap energy determination using UV-Vis DRS. In order to calculate the direct band gap of the prepared sample was used Tauc relation ${ }^{24}$ :

$(\alpha \mathrm{h} v)^{2}=\mathrm{B}\left(\mathrm{h} v-\mathrm{Eg}_{\mathrm{g}}\right)$ 
Where, $\alpha$ is the absorption coefficient, $h v$ is the photon energy, $\mathrm{B}$ is a constant value and $\mathrm{Eg}$ is the band gap energy. In equation (1), the absorption coefficient $(\alpha)$ is estimated by the following equation:

$\alpha=\frac{-1}{t} \ln T$,

where, $T$ is the transmittance value and $t$ is the thickness of the substance that is exposed to the UV-Vis irradiation. Based on the UV-Vis DRS, the plot of $(\alpha h v)^{\mathrm{n}} v s$. $\mathrm{h} v$ in $\mathrm{eV}$ was drawn. The band gap value was obtained by extrapolating the linear region of this curve. Fig. 5 shows the band gap energy plot with the UV-Vis absorption spectrum in the inset. The measured band gap was found to be $1.96 \mathrm{eV}$. This value indicates that this nanomaterial can be liable for the phtocatalytic activity in visible light domain.

\subsection{Photodegradation performance of AIS nanoparticles}

In order to study the phtodegradation performance of AIS nanoparticles, a series of the comparative experiments were carried out in purification of polluted water with 2,4-DCP under visible light irradiation. After $6 \mathrm{~h}$ of light irradiation time, the remained concentration of pollutant in aqueous solution was determined.

The removal efficiency of phtocatalytic activation was concluded from Eq. 3:

$$
\text { Removal efficiency }=\frac{C_{0}-C_{t}}{C_{0}} \times 100 \%
$$

where, $C_{0}$ is the initial 2,4-DCP concentration and $C_{t}$ is the pollutant concentration at time, $t$. All $C_{0}$ and $C_{t}$ values were determined by the maximum absorption at the wavelength of $248 \mathrm{~nm}$. The progress of the photocatalytic degradation of 2,4-DCP under visible light illumination was monitored by UV-Vis spectrophotometer. It was found that no degradation of 2,4-DCP was occurred in the absence of the photocatalyst under visible light irradiation. When the photocatalytic reaction was carried out in the dark, the removal efficiency of 3\% is resulted. In fact, it is originated from the partial adsorption of the organic molecules on the surface of photocatalyst particles. Degradation performance was significantly improved up to $95 \%$, when the $2,4-\mathrm{DCP}$ solution containing AIS nanoparticles was exposed to the visible light illumination (Fig. 6).

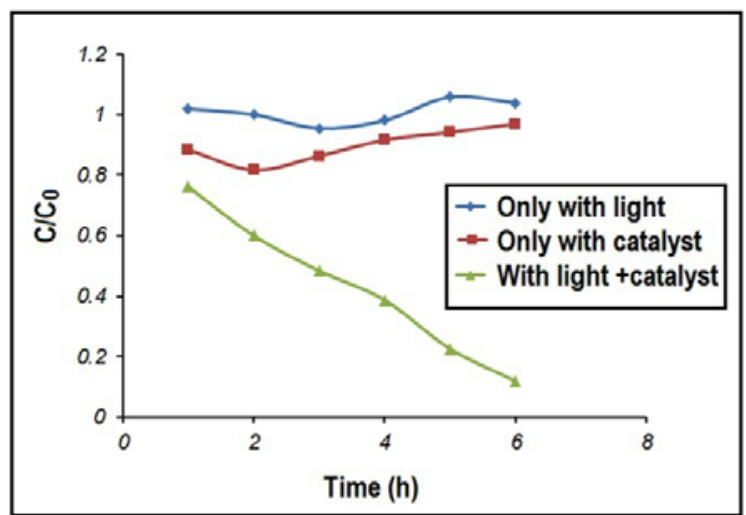

Fig. 6. Dependence of degradation of 2,4-DCP to the presence of light and catalyst

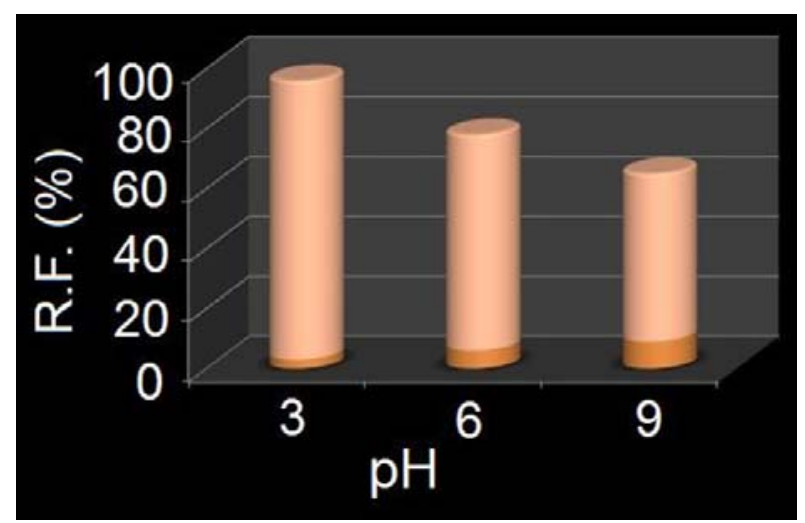

Fig. 7. The effect of initial solution $\mathrm{pH}$ on the degradation percentage of 2,4-DCP 


\subsubsection{Initial solution $p H$}

The effect of solution $\mathrm{pH}$ on the degradation of 2,4-DCP was investigated via adjusting solution $\mathrm{pH}$ to three values of 3,6 and 10 by $0.01 \mathrm{~mol} \mathrm{~L}^{-1} \mathrm{HCl}$ and $\mathrm{NaOH}$, respectively. Each of these runs was performed in the aqueous solutions of 2,4-DCP with $15 \mathrm{mg} \mathrm{L}^{-1}$ concentration $(50 \mathrm{~mL})$ containing $0.02 \mathrm{~g}$ AIS photocatalyst. Fig. 7 shows the degradation operation at different $\mathrm{pH}$ values. An enhanced removal percentage $(95 \%)$ was observed at $\mathrm{pH}=3$. This value declines to 81 and $43 \%$ at adjusted solution $\mathrm{pH}$ to 6 and 10, respectively. According to the mentioned results, the initial solution $\mathrm{pH}$ of 3 was selected for further studies.

\subsubsection{Initial 2,4-DCP concentration}

In order to study the influence of initial 2,4-DCP concentration on the removal efficiency, three initial pollutant concentrations i.e., 15,25 and $50 \mathrm{mg} \mathrm{L}^{-1}$ were examined. The results have been exhibited in Fig. 8. Due to increase the 2,4-DCP concentration from 15 to 25 and $50 \mathrm{mg} \mathrm{L}^{-1}$, the removal percentage of 2,4-DCP decreased from 95 to 80 and $70 \%$, respectively. This result can be ascribed to reduce the active sites on the catalyst surface. Meanwhile, the increase of pollutant concentration can decrease the light transmittance within suspension. Both of these reasons lead to decline the amount of hydroxyl radicals $\left(\mathrm{OH}^{\circ}\right)$ in medium and subsequently, the reduction of removal efficiency.

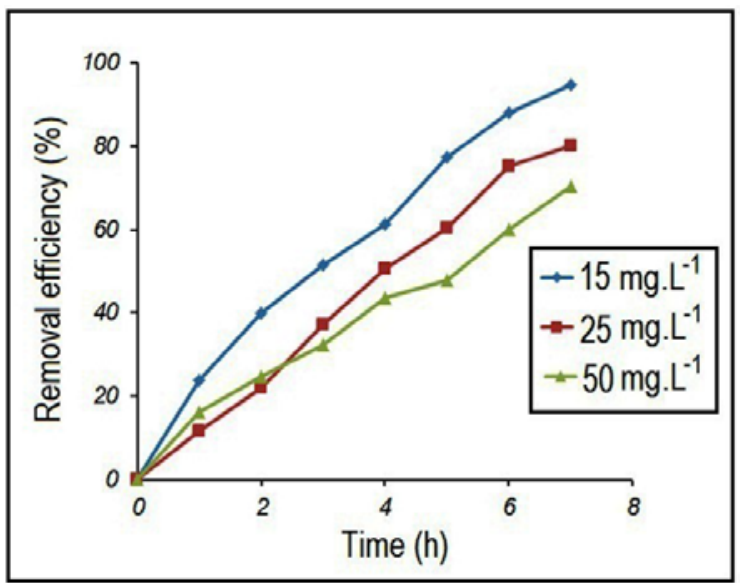

Fig. 8. Dependence of the removal efficiency to initial $2,4-\mathrm{DCP}$ concentration in various irradiation times

\subsubsection{Kinetic evaluation}

The scanning of photocatalytic reaction indicated that this operation can be described by the simplified kinetic model of pseudo first-order equation and its formula is given as:

$\operatorname{Ln}\left(\frac{C t}{C_{0}}\right)=-k t$

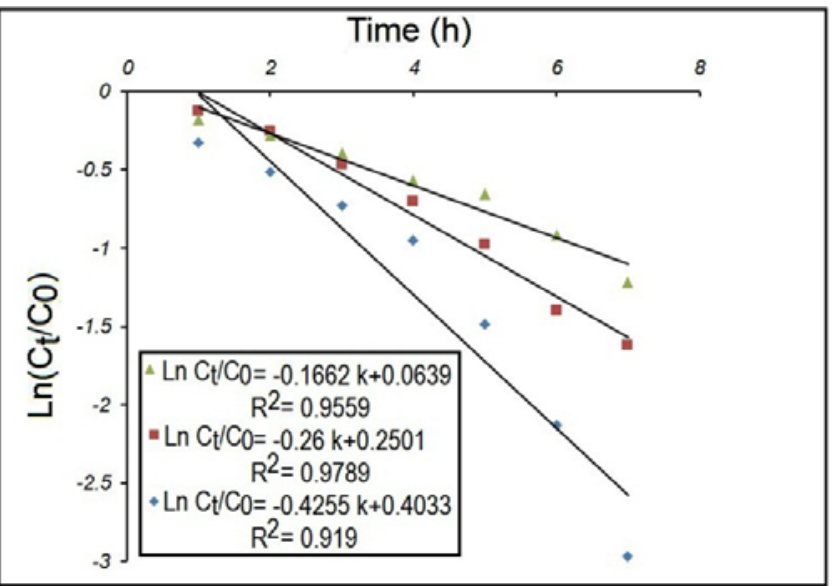

Fig. 9. The kinetics model of photodegradation performance of the $\mathrm{AgInS}_{2}$ nanoparticles, initial 2,4-DCP concentration of 15, 25 and $50 \mathrm{mg} \mathrm{L}^{-1}$, initial solution $\mathrm{pH}$ of 3 with the charge of fixed amount of photocatalyst $(0.02 \mathrm{~g})$

where, $C_{0}$ is the initial concentration $\left(\mathrm{mg} \mathrm{L}^{-1}\right)$ and $C_{t}$ is the concentration at time, $t$, the slope, $k$, is the apparent rate constant. By plotting $\operatorname{Ln}\left(\mathrm{C}_{t} / \mathrm{C}_{0}\right)$ vs. time, a linear relationship was observed for each of three experiments with different concentrations (Fig. 9). The results represented that the photocatalytic activity of AIS nanoparticles for removing the 2,4-DCP from aqueous solution follows the pseudo-first-order kinetic model. As seen in Fig. 9, the correlation coefficients $\left(\mathrm{R}^{2}\right)$ of pseudofirst-order model are $0.955,0.978$ and 0.919 for applied 2,4-DCP concentrations $\left(15,25\right.$ and $\left.50 \mathrm{mg} \mathrm{L}^{-1}\right)$, respectively. The all of three experiments show good fit to pseudo-first-order model, but the 
photocatalytic experiment with the 2,4-DCP concentration of $25 \mathrm{mg} \mathrm{L}^{-1}$ has a better fit than the other initial 2,4-DCP concentrations applied in this work.

\subsubsection{Photodegradation mechanism}

Generally, in a photocatalytic process, the excitation of electrons from valence band (VB) to conduction band (CB) and generation of electron-hole pairs take place on the surface of the photocatalyst by exposing to light irradiation. The exited electrons in $\mathrm{CB}$ and hole $\left(\mathrm{h}^{+}\right)$in VB are captured by oxygen molecules and $\mathrm{OH}^{-}$or $\mathrm{H}_{2} \mathrm{O}$ species dissolved in the suspension, respectively. The transfer of electron to oxygen molecule generates the superoxide anion and also, the presence of $\mathrm{h}^{+}$on VB band leads to produce hydroxyl radical $\left(\mathrm{OH}^{*}\right)$. These species depredate the organic pollutant adsorbed by the catalyst surface. The attack of produced radicals to aromatic carbon with its unpaired electron of 2,4-DCP molecule leads to break the $\pi$-bond of the aromatic ring, dechlorination and the $\mathrm{C}-\mathrm{O}$ bond formation. As a result, some by-products such as hydroquinone and quinoid structures maybe produced. Finally, degradation of 2,4-DCP is completed through forming of $\mathrm{CO}_{2}, \mathrm{H}_{2} \mathrm{O}$ and $\mathrm{Cl}^{-}$ as the final products ${ }^{1-3}$, as shown in Scheme 1.

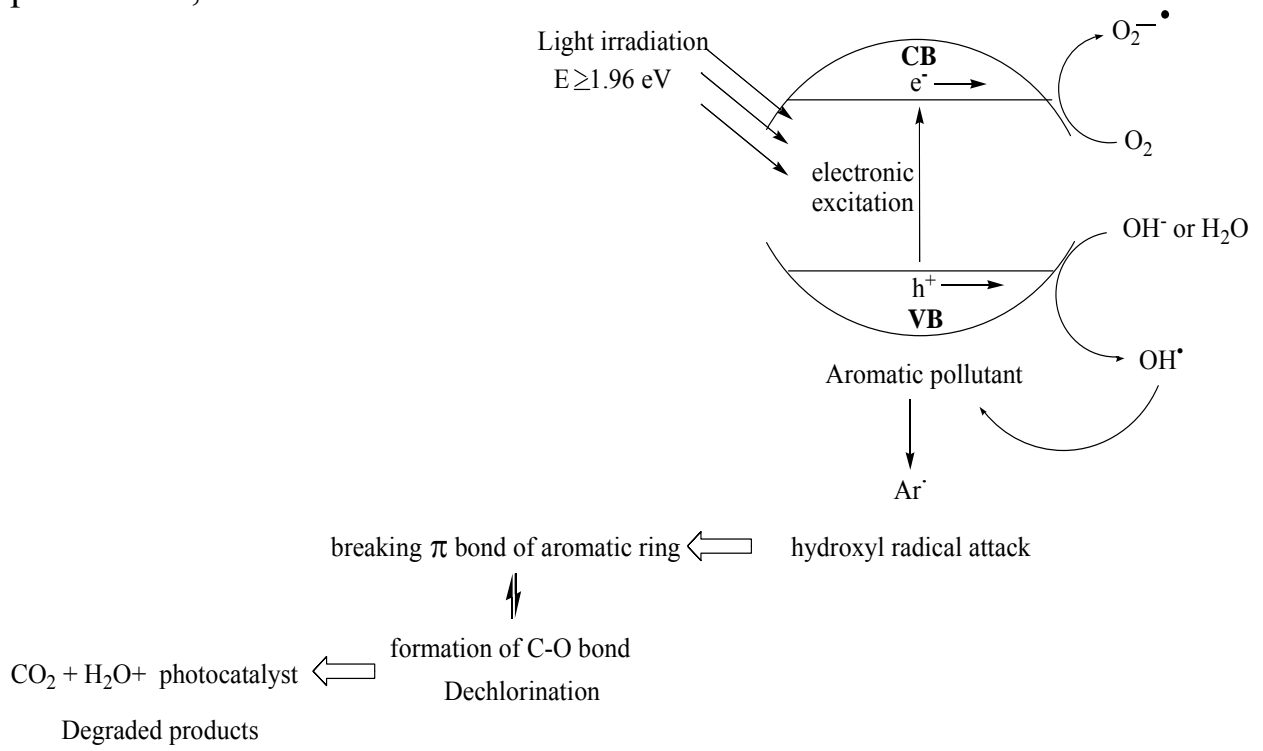

Scheme 1. The probable mechanism of phtocatalytic degradation of 2,4-DCP on the surface of $\mathrm{AgInS}_{2}$ nanoparticles under visible light irradiation

\section{Conclusions}

In summary, $\operatorname{AgInS} S_{2}$ nanoparticles were successfully prepared by a rapid and facile microwave heating technique using $\mathrm{AgNO}_{3}, \mathrm{InCl}_{3} .4 \mathrm{H}_{2} \mathrm{O}$ and thioacetamide as the starting materials. The structural and morphological studies confirmed the formation of a pure orthorhombic phase of $\mathrm{AgInS}_{2}$ nanoparticles with the average size of $70 \mathrm{~nm}$. The conformity of the band gap energy of this product $(1.96 \mathrm{eV})$ with the visible region of solar energy suggested that the obtained nanoparticles can be employed as an appropriate phtocatalyst in this domain. A series of phtocatalytic reactions for degradation of 2,4-DCP as a pollutant model under visible light illumination were carried out. Meanwhile, a comparative study of the effective parameters on the photocatalytic performance was studied. As a result, the degradation process of 2,4-DCP was dependent on $\mathrm{pH}$, and maximum removal efficiency $(95 \%)$ was obtained at $\mathrm{pH}$ of 3. It was found that the phtodegradation of 2,4-DCP on the AIS nanoparticles at different concentrations follows a kinetics model of pseudo-first-order rate. The results suggested the high capability of AIS nanoparticles for water and wastewater treatment under visible light irradiation.

\section{Acknowledgements}

The financial support of this study, by Iran University of Science and Technology and Iranian Nanotechnology Initiative, is gratefully acknowledged. 


\section{Experimental}

\section{Materials}

All reagents like indium (III) chloride tetrahydrate $\left(\mathrm{InCl}_{3} \cdot 4 \mathrm{H}_{2} \mathrm{O}\right)$, silver nitrate $\left(\mathrm{AgNO}_{3}\right)$, ethylene glycol, sodium dodecyl sulfate (SDS) and thioacetamide $\left(\mathrm{C}_{2} \mathrm{H}_{5} \mathrm{NS}\right)$ were purchased from Merck Co. and used without further purification.

\section{Characterization}

The X-ray diffraction (XRD) pattern was recorded by a JEOL diffractometer using $\mathrm{Cu}$ K $\alpha$ radiation (wavelength $=1.5418 \AA$ ). Scanning electron microscopy $(\mathrm{SEM})$ images were taken on a Philips XL30E SEM with gold coating. Transmission electron microscopy (TEM) images were measured on a ZEISS EM900 microscope working at $50 \mathrm{kV}$. Fourier transform infrared (FT-IR) spectrum was recorded on a Shimadzu- $8400 \mathrm{~S}$ spectrometer in the range of $400-4000 \mathrm{~cm}^{-1}$ using $\mathrm{KBr}$ pellets. The surface area of the product was obtained by using Brunauer-Emmett-Teller (BET) technique with Micromeritics (Gemini) in the range of relative pressures from 0.0 to 1.0. Before employing, the sample was degassed at $200{ }^{\circ} \mathrm{C}$ for $2 \mathrm{~h}$. In addition, the pore size distribution was determined from the desorption branch of the isotherm curve using the Barrett-Joyner-Halenda (BJH) model. The diffuse reflectance spectroscopy (DRS) was obtained using Shimadzu-UV-2550-8030 spectrophotometer in the range of 190-800 nm with slit width of $5.0 \mathrm{~nm}$ and light source with wavelength of $360.0 \mathrm{~nm}$ at room temperature.

\section{General procedure}

$\mathrm{AgInS}_{2}$ nanoparticles were prepared by microwave method ${ }^{20}$. In a summary, $58 \mathrm{mg}$ silver nitrate $\left(\mathrm{AgNO}_{3}\right), 100 \mathrm{mg}$ indium (III) chloride tetrahydrate $\left(\mathrm{InCl}_{3} \cdot 4 \mathrm{H}_{2} \mathrm{O}\right)$ and $51 \mathrm{mg}$ thioacetamide $\left(\mathrm{C}_{2} \mathrm{H}_{5} \mathrm{NS}\right)$ were dissolved in ethylene glycol, separately. After mixing the solutions, sodium dodecyl sulfate (SDS) as a surfactant was added to this solution. The resulting mixture was stirred and placed into a domestic microwave oven with the power of $900 \mathrm{~W}$ for $5 \mathrm{~min}$. The obtained precipitation was filtered and dried at $70{ }^{\circ} \mathrm{C}$ for $4 \mathrm{~h}$ in a vacuum oven.

\section{Photocatalytic activity measurements}

In this study, the photocatalytic behavior of the prepared AIS nanoparticles was evaluated via a probe reaction on the degradation of 2,4-dichlorophenol (2,4-DCP) in aqueous medium under visible light irradiation. A series of experiments were conducted to evaluate the influence of initial solution $\mathrm{pH}$ (with $\mathrm{pH}$ values of 3,6 and 10) and initial 2,4-DCP concentration $\left(15,25\right.$ and $\left.50 \mathrm{mg} \mathrm{L}^{-1}\right)$ on the photodegradation percentage. The fixed amount of photocatalyst $(0.02 \mathrm{~g})$ was charged into the reaction vessels. The solution was stirred for $1 \mathrm{~h}$ under dark conditions to establish the adsorptiondesorption equilibrium. At given intervals of irradiation $(60 \mathrm{~min}), 3 \mathrm{~mL}$ of suspension was taken away from the reaction vessels, centrifuged for $20 \mathrm{~min}$ to remove the remained catalyst from the solution. The visible light irradiation was carried out by a High-pressure mercury-vapor lamp (500W and $\lambda=546.8 \mathrm{~nm}$ ). The progress of photocatalytic reactions was monitored using the UV-Vis spectrophotometer at the wavelength of $315 \mathrm{~nm}$.

\section{References}

1. Choi J. W., Chung S. G., Cho K. Y., Baek K. Y., Hong S. W., Kim D. J., Lee S. H. (2012) Photocatalytic Degradation of Chlorophenol Compounds using Poly Aromatic Star Copolymer. Water Air Soil Pollut., 223, $1437-1441$ 
2. Jia J., Zhang S., Wang P., Wang H. (2012) Degradation of high concentration 2,4-dichlorophenol by simultaneous photocatalytic-enzymatic process using $\mathrm{TiO}_{2} / \mathrm{UV}$ and laccase. J. Hazard. Mater., 205-206, 150155.

3. Liu L., Chen F., Yang F., Chen Y., Crittenden J. (2012) Photocatalytic degradation of 2,4-dichlorophenol using nanoscale $\mathrm{Fe} / \mathrm{TiO}_{2}$. Chem. Eng. J., 181-182, 189-195.

4. Bayarri B., González O., Maldonado M. I., Giménez J., Esplugas S. (2007) Comparative Study of 2,4Dichlorophenol Degradation With Different Advanced Oxidation Processes. Trans. ASME., 129, 60-67.

5. Zhenxiang G. Li, C., Cheng S., Wei B., Xin Y. (2010) Photocatalytic degradation of 2,4- dichlorophenol using granular activated carbon supported $\mathrm{TiO}_{2}$. Desalination, 263, 107-112.

6. Mele G., Del Sole R., Vasapollo G., García-López E. (2003) Photocatalytic degradation of 4-nitrophenol in aqueous suspension by using polycrystalline $\mathrm{TiO}_{2}$ impregnated with functionalized $\mathrm{Cu}$ (II)-porphyrin or $\mathrm{Cu}(\mathrm{II})-$ phthalocyanine. J. Catal., 217, 334-342.

7. Mele G., Ciccarella G., Vasapollo G., Garcia-López E. (2002) Photocatalytic degradation of 4-nitrophenol in aqueous suspension by using polycrystalline $\mathrm{TiO}_{2}$ samples impregnated with $\mathrm{Cu}(\mathrm{II})$-phthalocyanine. App. Catal. B: Environ., 38, 309-319.

8. Wang C., Li J., Mele G., Yang G. M., Zhang F. X., Palmisano L., Vasapollo G. (2007) Efficient degradation of 4-nitrophenol by using functionalized porphyrin- $\mathrm{TiO}_{2}$ photocatalysts under visible irradiation. Appl. Catal. B: Environ., 76, 218-226.

9. Marais E., Klein R., Antunes E., Nyokong T. (2007) Photocatalysis of 4-nitrophenol using zinc phthalocyanine complexes. J. Mol. Catal. A: Chem., 261, 36-42.

10. Zhang W., Li D., Chen Z., Sun M., Li W., Lin Q., Fu X. (2011) Microwave hydrothermal synthesis of AgInS 2 with visible light photocatalytic activity. Mater. Res. Bull., 46, 975-982.

11. Friedmann D., Mendive C., Bahnemann D. (2010) $\mathrm{TiO}_{2}$ for water treatment: Parameters affecting the kinetics and mechanisms of photocatalysis. Appl. Catal. B: Environ., 99, 398-406.

12. Lei G., Xu M., Sun M., Fang H. (2006) Fabrication and characterization of nano $\mathrm{TiO}_{2}$ thin films at low temperature. Mater. Res. Bull., 41, 1596-1603.

13. Chen Y., Dionysiou D. D. (2006) Effect of calcination temperature on the photocatalytic activity and adhesion of $\mathrm{TiO}_{2}$ films prepared by the P-25 powder-modified sol-gel method. J. Mol. Catal. A: Chem., 244, 73-82.

14. Wada T., Kinoshita H., Kawata S. (2003) Preparation of chalcopyrite-type CuInSe $\mathrm{Cy}_{2}$ by non-heating process. Thin Solid Film, 431-432, 11-15.

15. Mao B., Chuang C. H., Wang J., Burda C. (2011) Synthesis and Photophysical Properties of Ternary I-III-VI $\mathrm{AgInS}_{2}$ Nanocrystals: Intrinsic versus Surface States. J. Phys. Chem. C., 115, 8945-8954.

16. Tian L., Vittal J. J. (2007) Synthesis and characterization of ternary AgInS $\mathrm{A}_{2}$ nanocrystals by dual- and multiplesource methods. New J. Chem., 31, 2083-2087.

17. Yoshino K., Komaki H., Kakeno T., Akaki Y., Ikari T. (2003) Growth and characterization of p-type AgInS 2 crystals. J. Phys. Chem. Solid, 64, 1839-1842.

18. Lopez M. O., Galan O. V., Candarilla F. C. (2003) Preparation of $\mathrm{AgInS}_{2}$ chalcopyrite thin films by chemical spray pyrolysis. Mater. Res. Bull., 38, 55-61.

19. Aissa Z., Bouzidi A., Amlouk M. (2010) Study of the I-V characteristics of $\mathrm{SnO}_{2}: \mathrm{F} / \mathrm{AgInS} \mathrm{S}_{2}(\mathrm{p}) / \mathrm{Al}$ Schottky diodes. J. Alloys Compd., 506, 492-495.

20. Tadjarodi A., Cheshmekhavar, A. H., Imani, M. (2012) Preparation of $\mathrm{AgInS}_{2}$ nanoparticles by a facile microwave heating technique; study of effective parameters, optical and photovoltaic characteristics. Appl. Surf. Sci., 263, 449-456.

21. Ge S., Shui Z., Zheng Z., Zhang L. (2011) A general microwave-assisted nonaqueous approach to nanocrystalline ternary metal chalcogenide and the photoluminescence study of $\mathrm{CoIn}_{2} \mathrm{~S}_{4}$. Opt. Mater., 33, 1174-1178.

22. Zhang Y., Feng S. B., Wang K., Yi X. H., Wang H. S., Pan Y. M. (2012) Water-solvent method for the synthesis of N-substituted and N-,4-disubstituted 1,8-naphthalimides under microwave irradiation. Synth. Commun., 42, 3042-3052.

23. Landry C. C., Lockwood J., Barron A. R. (1995) Synthesis of chalcopyrite semiconductors and their solid solutions by microwave irradiation. Chem. Mater., 7, 699-706.

24. Cui Y., Ren J., Chen G., Qian Y., Xie Y. (2001) A simple route to synthesize $\mathrm{MInS}_{2}(\mathrm{M}=\mathrm{Cu}, \mathrm{Ag})$ nanorods from single-molecule precursors. Chem. Lett., 236-237.

25. Gardner J. S., Shurdha E., Wang C., Lau L. D., Rodriguez R. G., Pak J. J. (2008) Rapid synthesis and size control of $\mathrm{CuInS}_{2}$ semi-conductor nanoparticles using microwave irradiation, J. Nanopart. Res., 10, 633-641.

26. Hu J. Q., Deng B., Tang K. B., Wang C. R., Qian Y. T. (2001) Preparation and phase control of nanocrystalline silver indium sulfides via a hydrothermal route, J. Mater. Res. 16, 3411-3415.

27. Nutek Inc. (2001) DOE-I basic design of experiments, Quality Engineering Seminar and Software Version: 080617, USA, Bloomfield Hills, MI.

28. Tadjarodi A., Imani M. (2011) Synthesis and characterization of $\mathrm{CdO}$ nanocrystalline structure by mechanochemical method, Mater. Lett. 65,1025-1027. 